\title{
ON APPROXIMATE AND PEANO DERIVATIVES
}

\author{
CLIFFORD E. WEIL ${ }^{1}$
}

Introduction. It has been known for some time that the inverse image of an open interval under a derivative either is empty or has positive measure (see [1] or [2]). Somewhat later it was shown that this property is also possessed by every approximate derivative and every $k$ th Peano derivative (see [4], [5] or [6]). At that time it was known that an approximate derivative is an ordinary derivative on an open dense set (see [3]), and likewise, a $k$ th Peano derivative is an ordinary $k$ th derivative on an open dense set (see [5]). This paper proves that these ordinary derivatives possess the above property on their sets of existence.

Definitions. Throughout, all functions are real valued functions defined on some fixed connected subset of the real line. Lebesgue measure on the line will be denoted by $u$.

Definition 1. A function $f$ has an approximate derivative $f_{\text {ap }}^{\prime}$ if for each $x$ in its domain there is a set $E$ whose density (computed relative to the domain of $f$ ) at $x$ is 1 such that

$$
\lim _{y \rightarrow x}(f(y)-f(x)) /(y-x)=f_{\mathrm{ap}}^{\prime}(x)
$$

where $y$ must lie in $E$.

Definition 2. A function $f$ has a $k$ th Peano derivative if for each $x$ in its domain there are numbers $f_{1}(x), \cdots, f_{k}(x)$ such that

$$
f(x+h)=f(x)+h f_{1}(x)+\cdots+\left(h^{k} / k !\right)\left(f_{k}(x)+\epsilon(x, h)\right)
$$

where $\lim _{h \rightarrow 0} \epsilon(x, h)=0$.

The function $f_{j}$ is called the $j$ th Peano derivative, $j=1, \cdots, k$. The basic properties of these two derivatives, including those mentioned in the introduction, may be found in [3]-[6].

Results. First it is shown that if the inverse image of an open interval under either of these derivatives is not empty, then it contains a point where the ordinary derivative (ordinary $k$ th derivative for the $k$ th Peano derivative) exists.

Lemma 1. Suppose $f$ has an approximate derivative. If $f_{\mathrm{ap}}^{\prime-1}(a, b)$ is not empty, then there is an $x$ in $f_{\mathrm{ap}}^{\prime-1}(a, b)$ where $f^{\prime}(x)$ exists. 1967.

Received by the editors August 14, 1967 and, in revised form, November 27, 1 This research supported in part by the National Science Foundation grant GP 7329. 
Proof. Suppose $(a, b)$ is an open interval such that $f_{\mathrm{ap}}^{\prime-1}(a, b)$ is not empty, but contains no points where the ordinary derivative exists. Let

$$
E_{a}=\left\{x: f^{\prime}(x) \text { exists and } f^{\prime}(x) \leqq a\right\}
$$

and

$$
E^{b}=\left\{x: f^{\prime}(x) \text { exists and } f^{\prime}(x) \geqq b\right\} .
$$

It will now be established that the interiors (relative to the domain of $f$ ) of the nondegenerate connected components of $E_{a}$ and $E^{b}$ form the complement of a perfect set. On the interior (computed relative to the domain of $f$ ) of a nondegenerate component of $E_{a}, f_{\text {ap }}^{\prime}$ is bounded above by $a$ and since an approximate derivative has the Darboux property (see [3]), the values of $f_{\text {ap }}^{\prime}$ at the endpoints of the component are no more than $a$. Hence, on the closure (computed relative to the domain of $f$ ) of the component, $f_{\mathrm{ap}}^{\prime}$ is bounded above by $a$, and thus, is $f^{\prime}$ there. It follows that two distinct components of $E_{a}$ cannot have a common endpoint. A similar statement can be made for $E^{b}$. Moreover, the Darboux property of $f_{\text {ap }}^{\prime}$ also implies that a component of $E_{a}$ cannot have an endpoint in common with a component of $E^{b}$.

Let $P$ be the perfect set whose complement is the union of the interiors of the nondegenerate components of $E_{a}$ and $E^{b}$. Since $f_{\mathrm{ap}}^{\prime}$ is a function of Baire class one, it must have a point of continuity in $P$ relative to $P$. Thus, the proof consists of verifying that if $f_{\mathrm{ap}}^{\prime-1}(a, b)$ contains no points where $f^{\prime}(x)$ exists, then $f_{\mathrm{ap}}^{\prime}$ has no point of continuity in $P$ relative to $P$. Let $x$ be in $P$ and let $I$ be any open interval containing $x$. There are points $y^{\prime}, z^{\prime}$ in $I$ with $f_{\text {ap }}^{\prime}\left(y^{\prime}\right) \geqq b$ and $f_{\text {ap }}^{\prime}\left(z^{\prime}\right) \leqq a$, for if there were, say, no such $y^{\prime}$, then $f_{\text {ap }}^{\prime}$ would be bounded above on $I$ and hence would be an ordinary derivative on $I$ (see [3]), and, by the assumption, $I$ would be contained in a component of $E_{a}$ contrary to $x$ in $I \cap P$. If $y^{\prime}$ is not in $P$, then it is in a component of $E^{b}$ and the endpoint, $y$, of that component lying between $x$ and $y^{\prime}$ is in $P \cap I$, with $f_{\mathrm{ap}}^{\prime}(y) \geqq b$. Likewise, there is a $z$ in $P \cap I$ with $f_{\mathrm{ap}}^{\prime}(z) \leqq a$. Thus, $x$ is the limit of two sequences $\left\{y_{n}\right\}$ and $\left\{z_{n}\right\}$ in $P$ such that $f_{\mathrm{ap}}^{\prime}\left(z_{n}\right) \leqq a$ and $f_{\mathrm{ap}}^{\prime}\left(y_{n}\right) \geqq b$. Hence $x$ can not be a point of continuity of $f_{\text {ap }}^{\prime}$ relative to $P$.

Lemma 2. Suppose $f$ has a kth Peano derivative $f_{k}$. If $f_{\mathbf{k}}^{-1}(a, b)$ is not empty, then there is an $x$ in $f_{\mathbf{k}}^{-1}(a, b)$ where $f^{(k)}(x)$ exists.

The proof is identical to that of Lemma 1 since all properties of $f_{\mathrm{ap}}^{\prime}$ used there are possessed by $f_{k}$ (see [5]). 
THEOREM 1. Suppose $f$ has an approximate derivative. If $f_{\mathrm{ap}}^{\prime-1}(a, b)$ is not empty, then $u\left(\left\{x: f^{\prime}(x)\right.\right.$ exists and lies in $\left.\left.(a, b)\right\}\right)>0$.

Proof. Let

$$
\begin{aligned}
E & =\left\{x: f^{\prime}(x) \text { exists and lies in }(a, b)\right\} \\
E_{a} & =\left\{x: f_{\mathrm{ap}}^{\prime}(x) \leqq a\right\}
\end{aligned}
$$

and

$$
E^{b}=\left\{x: f_{\mathrm{ap}}^{\prime}(x) \geqq b\right\} .
$$

By Lemma 1, $E$ is dense in itself, for if $x$ is in $E$ and $I$ an open interval containing $x$, then there is a point $y$ in $I, y \neq x$, with $f_{\mathrm{ap}}^{\prime}(y)$ in $(a, b)$ by the Darboux property of $f_{\mathrm{ap}}^{\prime}$. Take an interval $J$ contained in $I$, containing $y$ but not $x$. Then there is a $z$ in $J$ where $f^{\prime}(z)$ exists and lies in $(a, b)$. Thus, $\mathrm{Cl} E$ (closure being computed relative to the domain of $f$ ) is a perfect set. It will be shown that assuming $u(E)=0$ implies $\mathrm{Cl} E$ has no point of continuity of $f_{\text {ap }}^{\prime}$ relative to $\mathrm{Cl} E$.

Let $x$ be in $E$ and let $I$ be an open interval containing $x$. If $E_{a} \cap I$ is empty, then $f_{\mathrm{ap}}^{\prime}$ is bounded below on $I$, and hence is an ordinary derivative on $I$. Thus, $u(E \cap I)>0$ contrary to assumption. Thus $E_{a} \cap I$ is not empty nor is $E^{b} \cap I$. Now let $x$ be in $\mathrm{Cl} E$, and let $I$ be an open interval containing $x$. There is an $x^{\prime}$ in $I \cap E$, and consequently there is a $y^{\prime}$ in $I \cap E^{b}$ and $z^{\prime}$ in $I \cap E_{a}$. By the Darboux property for $f_{\mathrm{ap}}^{\prime}$ there is a $y^{\prime \prime}$ in $I$ with $f_{\mathrm{ap}}^{\prime}\left(y^{\prime \prime}\right)<b$ but as close to $b$ as desired, and there is a $z^{\prime \prime}$ in $I$ with $f_{\mathrm{ap}}^{\prime}\left(z^{\prime \prime}\right)>a$ but as close to $a$ as desired. Finally, by Lemma 1 , there is $y$ in $I$ where $f^{\prime}(y)$ exists, $f^{\prime}(y)<b$ but as close to $b$ as desired, and there is $z$ in $I$ where $f^{\prime}(z)$ exists, $f^{\prime}(z)>a$ but as close to $a$ as desired. Thus, $y$ and $z$ belong to $E$. Hence it is possible to construct two sequences $\left\{y_{n}\right\},\left\{z_{n}\right\}$ in $E$ converging to $x$ such that $\left\{f^{\prime}\left(y_{n}\right)\right\}$ converges to $b$ and $\left\{f^{\prime}\left(z_{n}\right)\right\}$ converges to $a$. Thus $x$ is not a point of continuity of $f_{\text {ap }}^{\prime}$ relative to $\mathrm{Cl} E$.

Theorem 2. If $f$ has a kth Peano derivative, and if $f_{\mathbf{k}}^{-1}(a, b)$ is not empty, then

$$
u\left(\left\{x: f^{(k)}(x) \text { exists and lies in }(a, b)\right\}\right)>0 .
$$

Again the proof is the same as that of Theorem 1 for the same reason.

\section{BiBLIOGRAPHY}

1. J. A. Clarkson, A property of derivatives, Bull. Amer. Math. Soc. 53 (1947). 124-125. 
2. A Denjoy, Sur une propriêté des fonctions dérivês, Enseignement Math. 18 (1916), 320-328.

3. C. Goffman and C. J. Neugebauer, On approximate derivatives, Proc. Amer. Math. Soc. 11 (1960), 962-966.

4. S. Marcus, On a theorem of Denjoy and on approximate derivatives, Monatsh. Math. 66 (1962), 435-440.

5. H. W. Oliver, The exact Peano derivative, Trans. Amer. Math. Soc. 76 (1954), $444-456$.

6. C. E. Weil, On properties of derivatives, Trans. Amer. Math. Soc. 114 (1965), 363-376.

Michigan State University 